\title{
Calibration of ionization chambers in air kerma length
}

\author{
François O Bochud, Mihail Grecescu and Jean-François Valley \\ Institute of Applied Radiophysics, Grand-Pré 1, CH-1007 Lausanne, Switzerland \\ E-mail: francois.bochud@inst.hospvd.ch
}

Received 27 April 2001, in final form 2 July 2001

Published 22 August 2001

Online at stacks.iop.org/PMB/46/2477

\begin{abstract}
Pencil ionization chambers are employed for the direct determination of the computed tomography dose index (CTDI), which is used for patient dosimetry in CT examinations. The chambers are calibrated in air kerma length in a reference x-ray field. The precision of calibration is influenced by several factors which are investigated in the present work. It is shown that the best irradiation length is $50 \%$ of the chamber rated length. The reading must be corrected for a residual signal due to the irradiation of the sensitive chamber volume by scattered radiation. The origin of this radiation is identified mainly at the edges of the diaphragm limiting the useful field. The residual signal is determined by a linear fit of measurements performed at minimum of three diaphragm apertures. The optimum geometric conditions are also discussed.
\end{abstract}

\section{Introduction}

Various national surveys concerning the collective dose received by the population during radiological examinations show that about one-third of the total dose is due to computed tomography (CT) (Schmidt 1999). This result implies that accurate dosimetry should be performed at CT facilities.

The dose descriptor most widely used in CT is the computed tomography dose index (CTDI) defined as follows (EUR 16262 1997):

$$
\text { CTDI }=\frac{1}{T} \int_{-\infty}^{\infty} D(z) \mathrm{d} z
$$

where $D(z)$ is the single scan dose profile along a line parallel to the rotation axis $(z)$ and $T$ is the nominal section thickness (full width at half maximum of the sensitivity profile). The integrand has the dimension dose by length, expressed in Gy $\mathrm{m}$ or usually in $\mathrm{mGy} \mathrm{cm}$. Other dose indices are defined by a similar formalism but with different integration limits and/or materials in which the measurement is performed (EUR 16262 1997, Nagel 2000).

The integral may be experimentally evaluated by different types of detectors: radiographic film, array of thermoluminescence dosimeters, array of silicon detectors, pencil 
ionization chamber. The pencil chamber has an elongated cylindrical geometry with a typical length of 10 to $15 \mathrm{~cm}$ and an external diameter of about $9 \mathrm{~mm}$. It is filled with air vented to the atmosphere. When the dose profile is not required, a calibrated pencil chamber directly provides the value of the integrated dose length product. The integration is obviously limited to the sensitive length of the ionization chamber. A few publications (Suzuki and Suzuki 1978, Pavlicek et al 1979, Poletti 1984, IEC 61674 1997, Cross 1998) deal with the determination of the pencil chamber characteristics; the calibration procedure is only briefly mentioned.

However, this calibration is not straightforward because the sensitive volume of the pencil chamber extends over a much larger axial length than for conventional diagnostic or radiotherapy ionization chambers. The calibration is performed in air in a uniform x-ray field with known air kerma rate; the calibrated quantity is the air kerma length. Two strategies are possible:

- Irradiation of a well defined fraction of the useful volume of the chamber; in this case, the remaining volume is sensitive to scattered radiation and contributes to the chamber signal. The situation is similar to the stem effect for conventional ionization chambers, but on a larger scale. A suitable correction has to be introduced.

- Irradiation of the whole sensitive volume of the pencil chamber, thus eliminating the influence of external scattered radiation. Unfortunately, as will be shown later, the limits of the sensitive volume are not precisely known, as they do not always coincide with the markers on the chamber body.

In the absence of a general agreement concerning the calibration conditions, various solutions are favoured by different people according to their knowledge. Metrologists as well as some manufacturers believe that by irradiating the whole sensitive volume of the pencil chamber a mean calibration factor may be determined, averaged over the small but unavoidable nonuniformity of the sensitivity along the chamber length. CT users think that the calibration should be performed by irradiating a length of 1 to $2 \mathrm{~cm}$, representing the most frequent section thickness. The International Electrotechnical Commission (IEC) recommends performing the calibration by irradiating $50 \%$ of the rated length, the chamber centre being positioned in the central axis of the beam (IEC 61674 1997).

The purpose of the present work is to investigate the relative merits of these approaches, the factors affecting their precision and to propose an optimal procedure for the calibration in air kerma length of the pencil ionization chambers.

\section{Material and method}

\subsection{Materials}

Two dosimeters for the determination of the air kerma length have been calibrated:

- a PTW Freiburg electrometer UNIDOS 10001 with an ionization chamber type 77336 (rated length $15 \mathrm{~cm}$ );

- a Radcal Corporation radiation monitor model 9015 with an ionization chamber model 10X5-3CT (rated length $10 \mathrm{~cm}$ ).

The calibration in air kerma of the x-ray beam was performed by a Nuclear Enterprises secondary standard consisting of an NE 2561 ionization chamber and of an NE 2560 electrometer. It has been calibrated against primary standards at the National Physical Laboratory (UK).

The irradiations were performed on a Philips MG 324 x-ray unit. According to IEC 61674 (1997), the reference radiation qualities (IEC 61267 1994) for testing dosimeters 
for measurement of air kerma length are RQR 9 (direct beam) and RQA 9 (attenuated beam). In the present calibration study the RQR 9 radiation quality $(120 \mathrm{kV}$, total filtration $2.5 \mathrm{~mm} \mathrm{Al}$, half-value layer (HVL) $4.7 \mathrm{~mm} \mathrm{Al}$ ) has mainly been used because of its larger air kerma rate. At the minimum distance to the focus $(75 \mathrm{~cm})$ the air kerma rate was $6.2 \mathrm{mGy} \mathrm{s}^{-1}$, leading to recombination charge losses less than $0.1 \%$ in the specified pencil chambers according to the manufacturer's specification. The results have also been checked with the RQA radiation quality $(120 \mathrm{kV}$, total filtration $42.5 \mathrm{~mm} \mathrm{Al}, \mathrm{HVL} 11.5 \mathrm{~mm} \mathrm{Al})$; its air kerma rate is 30 times lower than that of the RQR 9 radiation quality.

The ionization chambers were irradiated with their axis perpendicular to the anodecathode axis of the x-ray tube in order to avoid the non-uniformity due to the heel effect. The $\mathrm{x}$-ray field was shaped by different lead collimators with a circular or rectangular aperture. The uniformity of the field within an axial cross section of the chambers was in all cases better than $99.5 \%$ as determined by film exposure and by scanning with an NE 2571 ionization chamber.
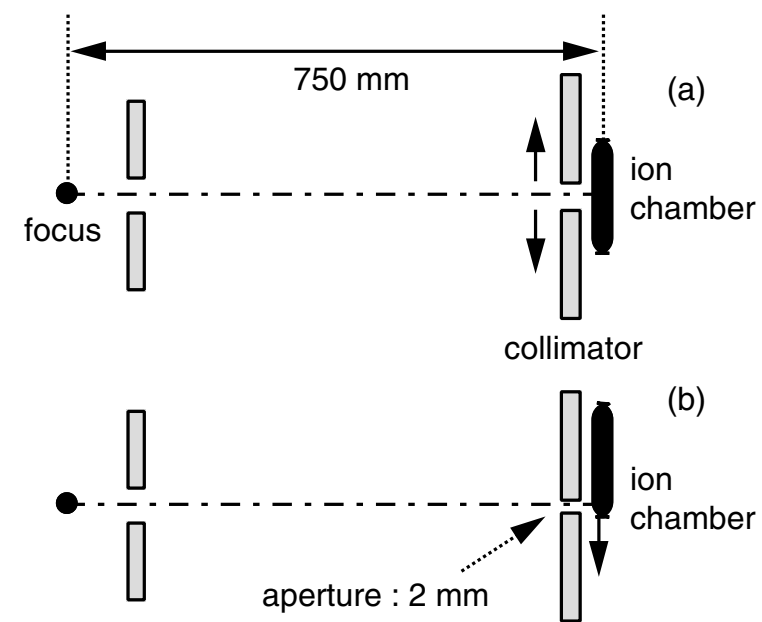

Figure 1. Geometry of the measurements performed on pencil ionization chambers: $(a)$ air kerma length calibration; $(b)$ determination of the spatial uniformity of the response.

A special irradiation stand was manufactured in order to irradiate various chamber volumes up to the rated length for calibration and to determine the spatial uniformity of the response (figure 1).

The length of the irradiated volume of the pencil chamber was varied by adjusting the aperture of a rectangular lead diaphragm positioned $42 \mathrm{~mm}$ in front of the chamber. Both aperture and chamber centres were situated on the beam axis. In order to achieve the desired field uniformity $(99.5 \%)$ at the maximum rated length of $15 \mathrm{~cm}$, the pencil chamber was placed at $150 \mathrm{~cm}$ from the focus. A circular field with a diameter of $25 \mathrm{~cm}$ (at $50 \%$ of the air kerma rate on the beam axis) was used.

On the basis of the results obtained in the first part of the present work, a different irradiation geometry was subsequently used (see section 3.3). The diaphragm has been placed close to the chamber in order to ensure sharp edges of the radiation field with negligible penumbra. This condition is very important for the precision of the calibration, as the uncertainty on the irradiated length $L$ (equation (3)) is a significant component of the total uncertainty (table 4). The geometry is quite different from that used in the calibration of radiotherapy ionization chambers or HVL determination, where the beam-shaping items are placed no closer than half 
the distance from the focal spot to the detector in order to avoid scattered radiation. The close geometry used in the present work has introduced scattered radiation and the proper correction of its effect is discussed below.

\subsection{Methods}

The calibration in air kerma length of the pencil chambers has been carried out in two steps, based on the use of a stable transmission ionization chamber as the beam monitor:

(a) The air kerma per unit monitor charge in reference ambient conditions $\left(K_{a} / Q_{\text {mon }}\right)_{r}$ has been determined with the secondary standard

$$
\left(\frac{K_{a}}{Q_{\mathrm{mon}}}\right)_{r}=N_{k}\left(\frac{R_{\mathrm{ss}}}{Q_{\mathrm{mon}}}\right) \frac{T_{\mathrm{ss}}}{T_{\mathrm{mon}}}
$$

where $N_{k}$ is the secondary standard calibration factor, $R_{\mathrm{ss}}$ its reading and $T_{\mathrm{ss}}$ and $T_{\mathrm{mon}}$ the temperatures of the secondary standard chamber and of the monitor.

(b) The pencil chamber reading per unit monitor charge in reference ambient conditions $\left(R / Q_{\text {mon }}\right)_{r}$ has been determined by placing the centre of the chamber at the position of the secondary standard reference point on the beam axis.

The pencil chamber reading has been corrected for scattered radiation, yielding a new ratio $\left(R_{c} / Q_{\text {mon }}\right) r$; the correction method will be presented later (section 3.1). The air kerma length calibration factor $N_{\mathrm{KL}}$ is calculated by the relation

$$
N_{\mathrm{KL}}=L \frac{\left(K_{a} / Q_{\mathrm{mon}}\right)_{r}}{\left(R_{c} / Q_{\mathrm{mon}}\right)_{r}}
$$

where $L$ is the irradiated length of the pencil chamber. This quantity was evaluated in the plane of the chamber axis by exposing a radiographic film. The uncertainty of about $0.2 \mathrm{~mm}$ is determined by the penumbra.

Some quantitative radiation measurements were also performed with radiographic films, as will be shown later (section 3.2). A fine-grain film, Kodak X-Omat V, exposed without an intensifying screen was used. Its absolute sensitometric curve (net optical density versus air kerma) was experimentally determined at the RQR 9 radiation quality using a standard procedure to avoid the film non-reciprocity effect. All films (for the sensitometric curve and for radiation measurements) were developed simultaneously. The readings were performed by digitizing the films with a precision optical scanner model Tango ${ }^{\mathrm{TM}}$ (Heidelberg Co.).

The spatial uniformity of response was measured by displacing the pencil chamber along its entire length in steps of $4 \mathrm{~mm}$ behind a $2 \mathrm{~mm}$ wide aperture delimited by two lead plates. The aperture is situated on the beam axis and the chamber extremities are displaced up to $\pm 15 \mathrm{~cm}$ with respect to this axis, the body of the chamber being shielded against the radiation by lead plates, except for the $2 \mathrm{~mm}$ aperture (figure $1(b)$ ). The scan starts at one extremity of the chamber. These conditions required the construction of a rather voluminous irradiation stand.

\section{Results and discussions}

\subsection{Calibrations performed at different lengths of the irradiated chamber volume}

The length of the irradiated volume of the pencil chamber was varied by incrementing in steps of $1 \mathrm{~cm}$ the diaphragm aperture from $1 \mathrm{~cm}$ to the rated length (10 and $15 \mathrm{~cm}$ respectively); the aperture corresponding to $50 \%$ of the rated length was also used. 


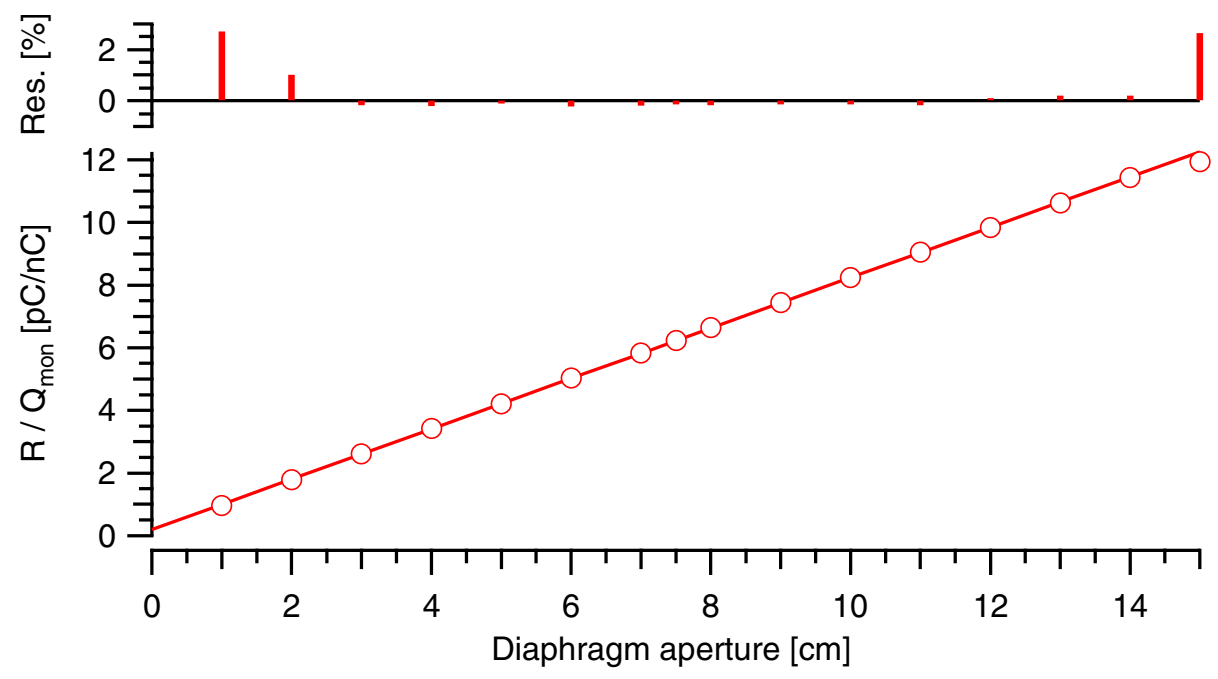

Figure 2. Normalized experimental readings of the PTW 77336 pencil ionization chamber versus diaphragm aperture and linear fit through points 1 to $14 \mathrm{~cm}$. The upper part of the graph shows the residual between the measured points and the fit.

The results, reported in detail below, were obtained with the PTW 77336 chamber. Similar measurements performed with the pencil chamber RADCAL Corp. model 10X5-3CT confirmed those results.

The normalized readings $\left(R / Q_{\text {mon }}\right)_{r}$ of the chamber PTW 77336 are plotted versus aperture width in figure 2 . The graph displays an excellent linearity except for one point (at $15 \mathrm{~cm}$ ); the linear regression through the points from 1 to $14 \mathrm{~cm}$ intercepts the ordinate axis at a positive value $\left(R_{0} / Q_{\text {mon }}\right)_{r}$. The origin of this residual signal will be explained later (section 3.2). A quantitative examination of the results shows that the differences between the experimental readings and the linear fit are less than $0.22 \%$ between 3 and $14 \mathrm{~cm}$; they increase up to $2.7 \%$ for the other three points.

The corrected reading $\left(R_{c} / Q_{\text {mon }}\right)_{r}$ was computed as follows

$$
\left(\frac{R_{c}}{Q_{\text {mon }}}\right)_{r}=\left(\frac{R}{Q_{\text {mon }}}\right)_{r}-\left(\frac{R_{0}}{Q_{\text {mon }}}\right)_{r} .
$$

The calibration factor $N_{\mathrm{KL}}$ calculated according to relation (3) for all the points is presented in figure 3. The results are extremely stable for aperture widths between 3 and $14 \mathrm{~cm}$; the difference between the mean of these values and that at $50 \%$ of the rated length is only $0.1 \%$. The calibration factors at the extreme apertures $(1,2$ and $15 \mathrm{~cm})$ are off the mean value by as much as 1.4 to $3.5 \%$.

The explanation of these results is the following:

- For small apertures the ratio of the corrected reading to the residual signal is less than 10 (4.1 at $1 \mathrm{~cm}$ and 8.4 at $2 \mathrm{~cm}$ ); this induces a greater uncertainty in the final result, such that the residual signal is only an average value provided by the linear fit; the measurements performed with films (section 3.2) suggest that its actual value might change slightly at small apertures.

- When irradiating the entire sensitive volume of the pencil chamber, the sensitivity decreases towards the extremities (section 3.4); the normalized reading is lower than expected and the calibration factor is pushed upward. 


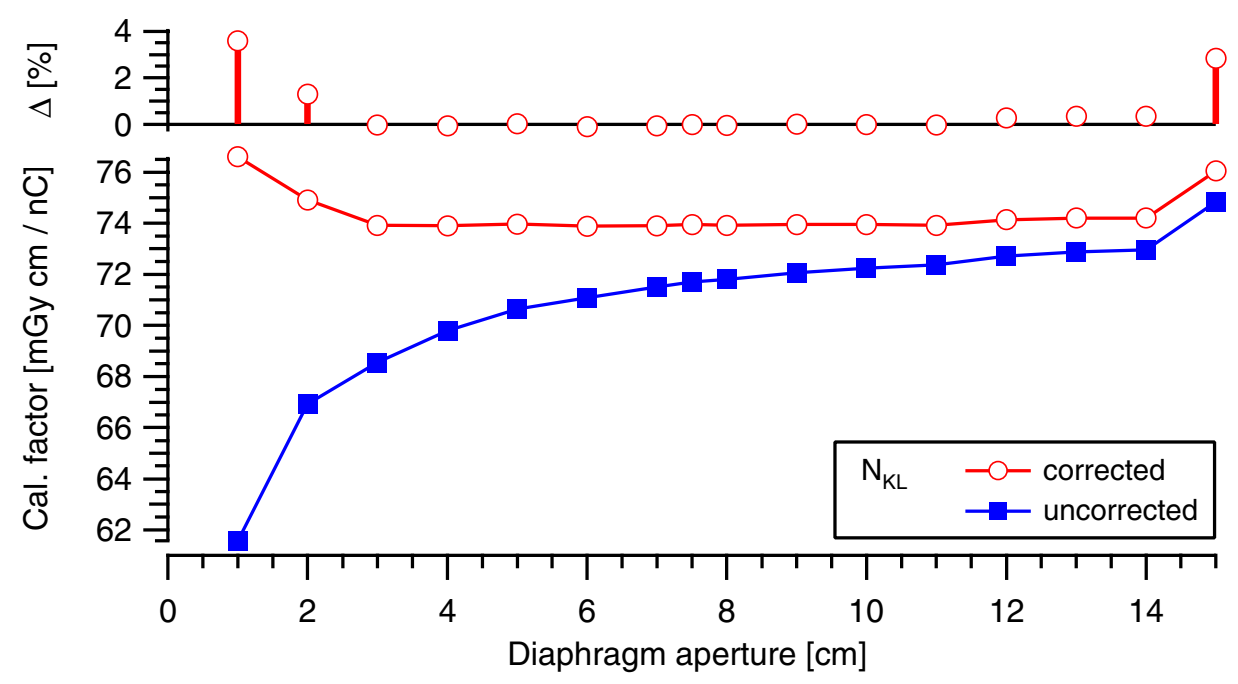

Figure 3. Air kerma length calibration factors of the PTW 77336 pencil ionization chamber versus diaphragm aperture: calculated with the corrected reading $\left(N_{\mathrm{KL}}\right)$ and with the uncorrected reading $\left(N_{\mathrm{KL}}^{b r}\right)$. The upper part of the graph shows the relative difference between the measured values and the measured value at a $7.5 \mathrm{~cm}$ aperture.

The previous procedure is time-consuming and therefore it is useful to investigate the consequences of performing the calibration by just one irradiation of a certain volume of the chamber. In this case there is no information about the residual signal and a calibration factor may be computed with relation (3) using the uncorrected reading $\left(R / Q_{\text {mon }}\right)_{r}$; this particular calibration factor is denoted $N_{\mathrm{KL}}^{b r}$. The results for the pencil chamber PTW 77336 are presented in figure 3 and they are unsatisfactory: the values continuously increase with the irradiated length and the difference between the extremes amounts to $21 \%$.

The results obtained so far show that of the different lengths of the irradiated chamber volume proposed at calibration (section 1), only that of the IEC standard (50\% of the rated length) leads to an accurate calibration factor provided that a correction for the residual signal is introduced.

The measurements performed with different diaphragm apertures are also interesting for the following reason: in the United States the dose index $\mathrm{CTDI}_{\text {Reg }}$ defined by the Center for Devices and Radiological Health $(\mathrm{CSDH})$ requires the integration over 14 slices, regardless of their thickness; for the determination of the $\mathrm{CTDI}_{\text {Reg }}$ in the case of a thin slice scan ( 1 to $5 \mathrm{~mm}$ ) the pencil chamber measurements must be adjusted by correction factors established by irradiating different lengths of the central exposed portion of the chamber (Rothenberg and Pentlow 1995).

\subsection{Origin of the residual signal}

The graph of figure 2 might suggest that the residual signal $\left(R_{0} / Q_{\text {mon }}\right)_{r}$ corresponds to the chamber reading with the lead diaphragm completely shut; in this case it should be due to the radiation scattered by the irradiation stand and by other objects in the room. Actually this assumption is wrong, as shown by the measurement of the pencil chamber reading with the lead diaphragm shut; the value is up to an order of magnitude lower than the residual signal deduced from the graph, depending on the shape and dimensions of the radiation field. This result points to another origin of the residual signal. 


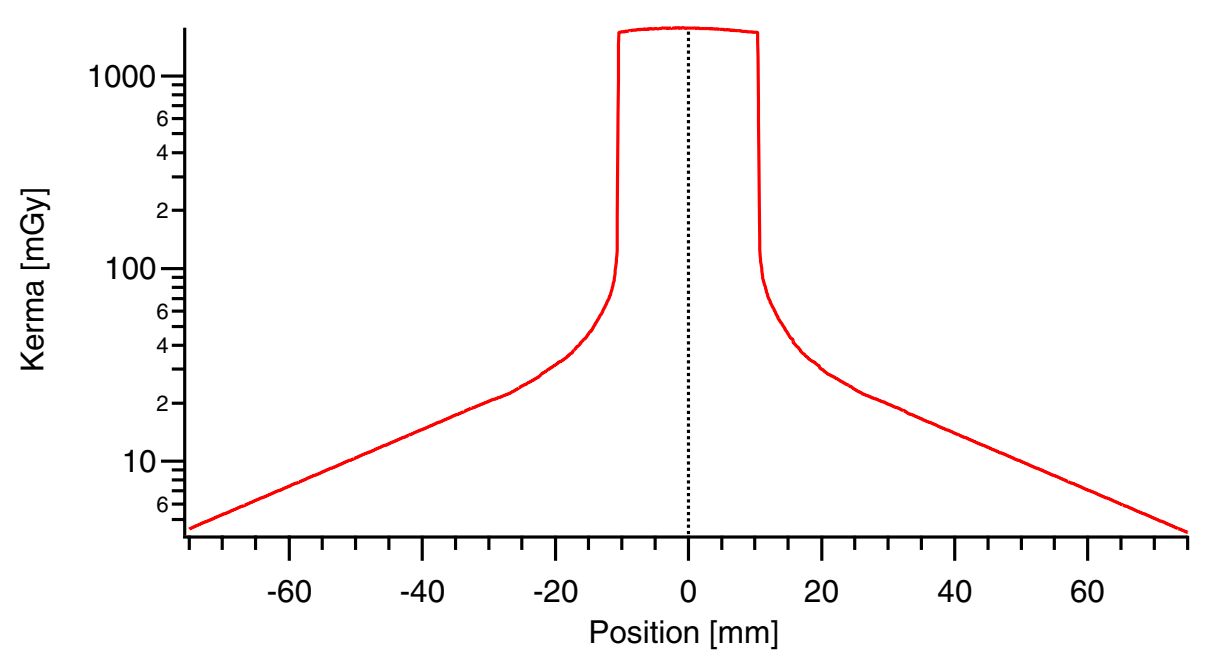

Figure 4. Distribution of air kerma along the pencil chamber axis determined with Kodak X-Omat V films. Diaphragm aperture $2 \mathrm{~cm}$.

In order to determine the radiation pattern at the position of the chamber, Kodak X-Omat V films were exposed in the vertical plane containing the chamber axis with a diaphragm aperture of $2 \mathrm{~cm}$. Because of the expected large range of kerma values and of the limited film latitude, four films were exposed with times of 13.5, 135, 430 and $1350 \mathrm{~s}$. The films were digitized and the optical density was converted to air kerma using the absolute sensitometric curve of the film. The normalization of the results was performed with the monitor chamber readings $\left(Q_{\text {mon }}\right)$ and eventually a unified set of data was obtained. The variation of the air kerma along the pencil chamber axis is represented in figure 4.

The examination of the films shows that the non-irradiated volume of the pencil chamber receives mainly the radiation scattered by the edges of the diaphragm; this is also visible in figure 4. This radiation is absent when the aperture is completely shut, which explains the previously mentioned difference between the chamber response measured in these conditions and the residual signal $\left(R_{0} / Q_{\text {mon }}\right)_{r}$.

The chamber response due to scattered radiation was evaluated by numerical integration of the curve in figure 4 between the edge of the radiation field and the chamber extremity. The results of the measurements performed by film and by the pencil chamber PTW 77336 are compared in table 1 .

The agreement between both methods is acceptable, taking into account the following possible sources of error:

- The non-reciprocity effect due to the difference between the film exposure times (up to a factor of 100).

- The conversion from optical density to air kerma using the film characteristic.

- The residual signal $\left(R_{0} / Q_{\text {mon }}\right)_{r}$ for the ionization chamber is a mean value in which large apertures have a predominant weight; the residual signal on film is measured with a $2 \mathrm{~cm}$ aperture and the integration interval is obviously longer, leading to a somewhat larger residual signal.

However, the very stable values of the calibration factor for apertures between 3 and $14 \mathrm{~cm}$ (figure 3 ) show that the mean value of the residual signal as determined by the linear regression 
Table 1. Comparison of air kerma length measurements by the pencil ionization chamber PTW 77336 and by film.

\begin{tabular}{llll}
\hline Quantity & Ion chamber & Film & $\begin{array}{l}\text { Relative } \\
\text { difference (\%) }\end{array}$ \\
\hline$K_{a} L(\mathrm{mGy} \mathrm{cm})$ & 3420 & 3650 & 6.7 \\
Residual signal/useful signal & 0.070 & 0.071 & 1.4 \\
\hline
\end{tabular}

Table 2. Comparison of signals in two geometries.

\begin{tabular}{llll}
\hline \multirow{2}{*}{$\begin{array}{l}\text { Focus to chamber } \\
\text { distance }(\mathrm{cm})\end{array}$} & Calibration field & \multicolumn{2}{c}{ Slope/(ordinate at origin) } \\
\cline { 3 - 4 } & dimensions (mm) & PTW 77336 & Radcal 10X5-3CT \\
\hline 75 & Rectangular 85 × & 23.5 & 47.4 \\
150 & Circular $\varnothing 160$ & 4.2 & 5.8 \\
\hline
\end{tabular}

is a good approximation in these cases. The explanation resides in the steep decrease of the scattered radiation away from the field edges: $95 \%$ of the integral value is concentrated within the first $2 \mathrm{~cm}$.

\subsection{Procedure for the calibration of ionization chambers in air kerma length}

The preceding results have shown that the main factors influencing the calibration of pencil chambers is air kerma length. On this basis a set of optimal conditions for calibration have been implemented.

As the calibration should be performed by irradiating $50 \%$ of the chamber rated length, the largest dimension of the uniform radiation field has been reduced by a factor of two; consequently the focus to chamber distance has also been decreased from $150 \mathrm{~cm}$ to $75 \mathrm{~cm}$. In order to reduce the components of scattered radiation, the dimensions of the x-ray field were limited to the minimum. A rectangular field of $8.5 \times 1.5 \mathrm{~cm}$ has been implemented; its uniformity is better than $99.5 \%$ as tested with a film. The improvement in the ratio (useful signal)/(residual signal) was estimated by the ratio slope/(ordinate at origin) in linear regressions such as that in figure 2 . The results are presented in table 2.

At $150 \mathrm{~cm}$ the ratios are of the same order and are comparable to the value 3.8 obtained by Pavlicek et al (1979) for the pencil chamber Radcal 20X5-103CT. At $75 \mathrm{~cm}$ and with the rectangular field, a significant improvement of the ratio is obtained. The difference between both chambers might be explained as follows: the investigated ratio and especially the ordinate at the origin are critically dependent on the position of the experimental points in the graph; they are closer to the linear fit for the 10X5-3CT chamber whose sensitivity has a more uniform spatial variation (section 3.4).

The calibration procedure developed previously (section 3.1) is reliable but too timeconsuming for a routine use. Nevertheless more than one measurement is necessary for the determination of the residual signal and a test was conducted using three measurements. The comparison was done between calibrations performed in the following conditions:

- PTW 77336 chamber: nine apertures (1 to $8 \mathrm{~cm}$ in steps of $1 \mathrm{~cm}$ plus $7.5 \mathrm{~cm}$ ) and three apertures $(3,5,7.5 \mathrm{~cm})$.

- Radcal 10X5-3CT chamber: eight apertures ( 1 to $8 \mathrm{~cm}$ in steps of $1 \mathrm{~cm}$ ) and three apertures $(3,5,7 \mathrm{~cm})$. 
Table 3. Comparison of calibration factors obtained in different conditions.

\begin{tabular}{lll}
\hline \multirow{2}{*}{$\begin{array}{l}\text { Number of apertures used } \\
\text { in the linear regression }\end{array}$} & \multicolumn{2}{c}{$N_{\mathrm{KL}}\left(\mathrm{mGy} \mathrm{cm} \mathrm{div}^{-1}\right)$} \\
\cline { 2 - 3 } & PTW 77336 & Radcal 10X5-3CT \\
\hline Three & 73.4 & 9.57 \\
Eight or nine & 73.3 & 9.59 \\
Relative difference (\%) & 0.14 & -0.21 \\
\hline
\end{tabular}

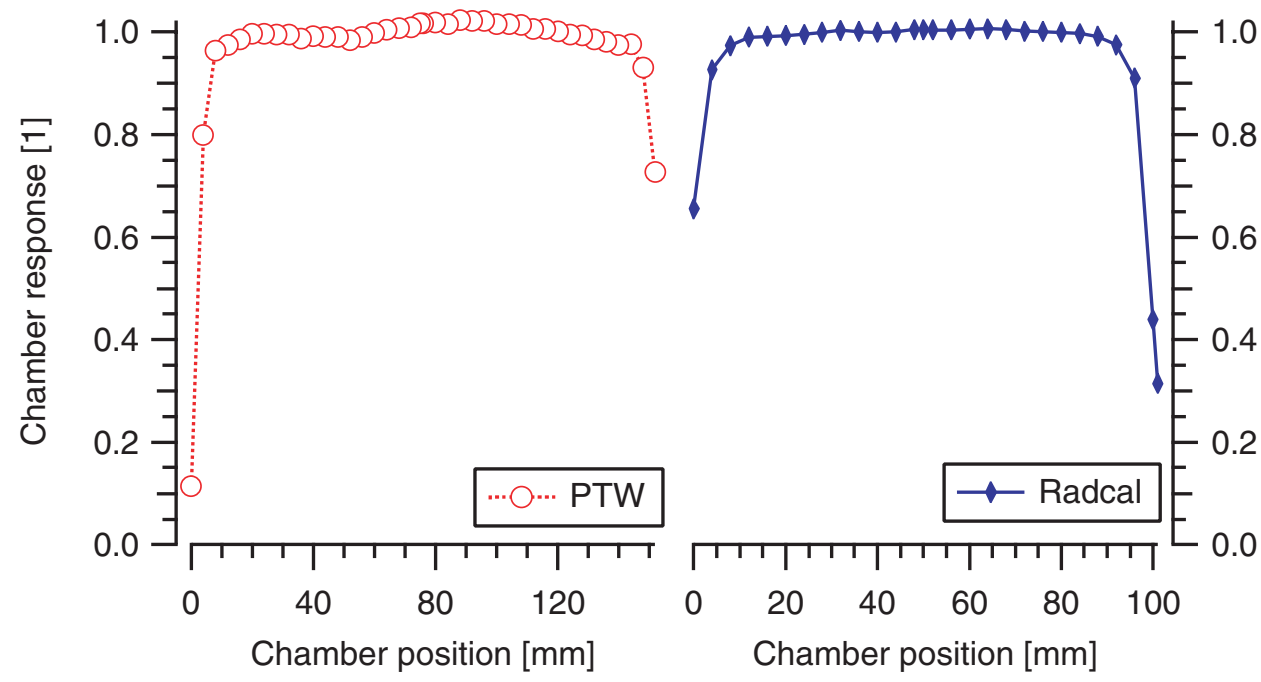

Figure 5. Spatial uniformity of response of PTW 77336 (rated length marks at 0 and $150 \mathrm{~mm}$ ) and Radcal 10X5-3CT (rated length marks at 0 and $100 \mathrm{~mm}$ ) pencil ionization chambers.

The results are presented in table 3. The agreement being well within the calibration uncertainty, the simplified procedure is acceptable.

The improvement of the ratio (useful signal)/(residual signal) in the new geometry (table 3) may suggest that the correction for the residual signal is no longer significant and that a single measurement at $50 \%$ of the rated length would be enough. The test of this hypothesis showed that the difference between the calibration factors $N_{\mathrm{KL}}^{b r}$ and $N_{\mathrm{KL}}$ is acceptable $(-0.1 \%)$ for the Radcal 10X5-3CT chamber but too large $(-0.6 \%)$ for the PTW 77336 chamber. This oversimplification of the procedure is actually pointless, because it implies knowledge of the residual signal before deciding to neglect it.

The established procedure was also applied to the calibration of the PTW 77336 chamber at the RQA 9 radiation quality; the calibration factor is $74.6 \mathrm{mGy} \mathrm{cm} \mathrm{div}^{-1}$. The difference between the values of $N_{\mathrm{KL}}$ at both radiation qualities is well within the uncertainty, suggesting that the energy response in this range (equivalent energies between 40 and $73 \mathrm{keV}$ ) is rather flat.

\subsection{Spatial uniformity of response of pencil ionization chambers}

The measuring procedure was described earlier (section 2.2). The irradiated volume being very small (aperture width $2 \mathrm{~mm}$ ) the following steps were taken to improve the ratio (useful signal)/(residual signal): reduction of the focus-chamber distance to the minimum possible 
Table 4. Estimated relative standard uncertainties in the calibration of pencil ionization chambers. Quantity $u$ is the combined uncertainty.

\begin{tabular}{|c|c|c|c|c|}
\hline \multirow[b]{2}{*}{ Term } & \multirow{2}{*}{$\begin{array}{l}\text { Type A } \\
(\%)\end{array}$} & \multicolumn{2}{|l|}{ Type B uncertainty } & \multirow{2}{*}{$\begin{array}{l}u \\
(\%)\end{array}$} \\
\hline & & Source & $(\%)$ & \\
\hline$N_{k}$ & & Calibration & & 0.55 \\
\hline$\left(R_{\mathrm{ss}} / Q_{\mathrm{mon}}\right)$ & 0.1 & Medium-term stability of instruments & 0.2 & 0.22 \\
\hline$T_{\mathrm{ss}}, T_{\mathrm{mon}}, T$ & & Calibration & & 0.03 \\
\hline \multirow[t]{2}{*}{$\left(K_{a} / Q_{\text {mon }}\right)_{r}$} & & Temperature correction & 0.6 & 0.68 \\
\hline & & Detect. position, transfer to RQR 9 rad. quality & 0.3 & \\
\hline \multirow[t]{2}{*}{$\left(R / Q_{\mathrm{mon}}\right)_{r}$} & 0.1 & Medium-term stability of instr., temp. correction & 0.4 & 0.66 \\
\hline & & Detector position, radiation field homogeneity & 0.5 & \\
\hline$\left(R_{0} / Q_{\text {mon }}\right)_{r}$ & - & Linear regression & 0.3 & 0.3 \\
\hline$L$ & 0.2 & Aperture width and penumbra & 0.9 & 0.92 \\
\hline$N_{\mathrm{KL}}$ & & & & 1.35 \\
\hline
\end{tabular}

value; reduction of the radiation field size to the minimum necessary. The results for both pencil chambers studied are presented in figure 5 .

The standard IEC 61674 (1997) specifies that the limits of the rated length shall be clearly marked and that over the rated length the spatial uniformity of response shall not vary by more than $\pm 3 \%$. The last requirement is not satisfied for either of the tested units: the uniformity is within the specification along most of the chamber length, but drops a few $\mathrm{mm}$ before the marks on the chamber body. The consequence of this non-uniformity was discussed in section 3.1. This result obtained for one particular unit of each chamber model does not imply that all chambers have the same problem; however, a long period of measurement is necessary in order to assess that any particular chamber behaves correctly.

\subsection{Estimation of uncertainties}

The estimation of type A and type B standard uncertainties (ISO 1995) for the terms of equations (2), (3) and (4) are presented in table 4 together with the main sources of uncertainty. The expanded uncertainty (coverage factor 2) of the pencil chamber calibration factor is typically $2.7 \%$.

\section{Conclusion}

The factors influencing the calibration in air kerma length of pencil ionization chambers have been investigated and useful practical conclusions deduced.

In order to obtain accurate results, the calibration factor should be determined by irradiating $50 \%$ of the rated length of the chamber, in agreement with IEC standard 61674 (1997).

The sensitive chamber volume outside the primary $\mathrm{x}$-ray field contributes to the reading by detecting scattered radiation. Most of that radiation originates at the edges of the diaphragm limiting the primary field.

The chamber reading must be corrected for the residual signal due to scattered radiation before computing the calibration factor. The residual signal is determined as the ordinate axis intercept of a linear fit through chamber readings taken at three different diaphragm apertures between about 3 and $8 \mathrm{~cm}$.

The calibration geometry is determined by several requirements: the pencil chamber axis is perpendicular to the anode-cathode axis of the x-ray tube because of the heel effect; the 
scattered radiation is reduced by using a rectangular radiation field; its size along the chamber axis should be slightly greater than $50 \%$ of the chamber rated length and slightly larger than the chamber diameter along the perpendicular direction; the uniformity of the radiation field should be better than $99.5 \%$ across this area; this condition determines the minimum focus to chamber distance.

\section{Acknowledgments}

The authors wish to thank Dr Renate Moning of the Swiss Federal Office of Metrology and Accreditation (METAS) for helpful discussions and for lending the PTW equipment.

\section{References}

Cross P 1998 Determination of CT scanner radiation output: correction factors for partial irradiation of thimble and pencil ionisation chambers by collimated fan beams Australas. Phys. Eng. Sci. Med. 21 29-31

EUR 162621997 Quality Criteria for Computed Tomography (Luxembourg: European Commission)

IEC 612671994 International Standard: Medical Diagnostic X-ray Equipment-Radiation Conditions for use in the Determination of Characteristics (Geneva: International Electrotechnical Commission)

IEC 616741997 International Standard: Medical Electrical Equipment_Dosimeters with Ionisation Chambers and/or Semi-Conductor Detectors as used in X-ray Diagnosis Imaging (Geneva: International Electrotechnical Commission)

International Organization for Standardization (ISO) 1995 Guide to the Expression of Uncertainty in Measurement (Geneva: ISO in the name of BIPM, IEC, IFCC, ISO, IUPAP, OIML)

Nagel H D 2000 Radiation Exposure in Computed Tomography-Fundamentals, Influencing Parameters, Dose Assessment, Optimisation, Sanner Data, Terminology ed H D Nagel (Frankfurt: COCIRI) pp 5-13

Pavlicek W, Horton J and Tuco R 1979 Evaluation of the MDH Industries, Inc. pencil chamber for direct beam CT measurements Health Phys. 37 773-4

Poletti J L 1984 An ionization chamber based CT dosimetry system Phys. Med. Biol. 29 725-31

Rothenberg L and Pentlow K 1995 CT dosimetry and radiation safety Medical CT and Ultrasound: Current Technology and Applications ed L W Goldman and J B Fowlkes (Madison, WI: Advanced Medical Publishing) pp 532-4

Schmidt Th 1999 Zur Situation der Strahlenexposition bei CT Strahlenexposition in der Computertomographie ed H D Nagel (Hamburg: Fachverband Elektromedizinische Technik im ZVEI) pp 1-3

Suzuki A and Suzuki M N 1978 Use of a pencil-shaped ionisation chamber for measurement of exposure resulting from a computed tomography scan Med. Phys. 5 536-9 